\title{
Best Practices and Protocols in Mössbauer Spectroscopy
}

Fernande Grandjean* and Gary J. Long*

Department of Chemistry, Missouri University of Science and Technology, University of Missouri, Rolla, Missouri 65409-0010, United States

\section{Contents}

\section{CHECK-LIST FOR AUTHORS AND REVIEWERS OF PAPERS REPORTING MÖSSBAUER SPECTRAL RESULTS}

The following one-page numbered check-list provides guidelines for writing and reviewing the Mössbauer spectral sections of a paper and guidelines for spotting missing or inaccurate presentation of the results. Authors of such papers may save themselves much time and effort by using this checklist. All of the following problems are discussed in the paper noted aabove and have been repeatedly observed in the current literature. Most items apply specifically to iron-57 Mössbauer spectra but many also apply to results reported for other Mössbauer-active nuclides.

A one page numbered check-list follows on the next page. 


\section{CHECK-LIST FOR AUTHORS AND REVIEWERS OF PAPERS REPORTING MÖSSBAUER SPECTRAL RESULTS}

1. The source matrix, usually rhodium metal for iron-57 spectra, must be reported.

2. The temperature of the source, usually $295 \mathrm{~K}$, and of all measured spectra must be reported, preferably in Kelvin.

3. The isomer shift reference standard, usually $\alpha$-iron for iron-57 spectra at $295 \mathrm{~K}$, must be reported.

4. The line width of the calibration spectrum should be reported.

5. If the spectrometer drive is operating in a sinusoidal mode this must be indicated. Also whether or not the velocity scale was linearized in any spectral plot must be indicated.

6. The thickness of all absorbers, usually in $\mathrm{mg} / \mathrm{cm}^{2}$ of either natural iron or iron-57 must be reported.

7. The line width, $\Gamma$, usually the full width at half-maximum must be reported. The reason for multiple line widths should be explained.

8. A plot of at least one experimental Mössbauer spectrum and its fit must be reported.

9. Mössbauer spectral figures must have both axes correctly labeled. Usually for iron-57 spectra this would include Velocity, $\mathrm{mm} / \mathrm{s}$ and Percent Transmission. The velocity units should never be labeled as mm/sec. The scale of the Percent Transmission or Percent Absorption must be given or well defined. "Arbitrary Units", or "A. U." or nothing are never acceptable.

10. The velocity scale for iron -57 spectra should be symmetric, i.e., $\pm 4 \mathrm{~mm} / \mathrm{s}$ and not -2 to +4 $\mathrm{mm} / \mathrm{s}$.

11. Both the experimental spectral data points and their fit must be clearly visible.

12. The velocity scale must have a zero velocity that corresponds to the isomer shift reference standard.

13. In a figure showing the temperature dependence of Mössbauer spectra, the highest temperature should be at the top and the lowest temperature at the bottom.

14. All fitted spectral parameters including the line width must be reported, preferably in a table, with an uncertainty, typically the statistical uncertainty. These uncertainties should not be referred to as error bars. The table values must agree with those also quoted in the text and shown in any spectral plots.

15. An excessively large line width, i.e., larger than ca. $0.4 \mathrm{~mm} / \mathrm{s}$ must be justified or explained.

16. A full width at half-maximum of less than $0.195 \mathrm{~mm} / \mathrm{s}$ for iron-57 spectra violates the Heisenberg uncertainty principle and must never be reported. It is best to avoid reporting half width at half-maximum values.

17. In a magnetic spectrum the quadrupole splitting and quadrupole shift parameters must never be confused - they are different parameters.

18. The hyperfine field should be reported in $\mathrm{T}, \mathrm{kG}$, or $\mathrm{kOe}$, where tesla, $\mathrm{T}$, is preferable.

19. The total absorption area should be reported, typically in $(\% \varepsilon)(\mathrm{mm} / \mathrm{s})$ for iron-57 spectra.

20. Fractional area or percent area values for different components in a spectrum must be reported with their uncertainties.

21. All the appropriate units should be given in SI units with the correct abbreviations. Any $\mathrm{kcal} / \mathrm{mol}$ units should be given in $\mathrm{kJ} / \mathrm{mol}$.

22. If a spectral fit involves a large number of fitted parameters, the correlation coefficients between the refined parameters should be determined and any correlation values above ca. 0.7 should be reported. Fits correlation values above ca. 0.7 should be considered suspect.

23. Finally, the authors must use an English version of spell-check to check all spelling. The authors should not change "quadrupole splitting" to "quadruple splitting." 